\title{
White Electroluminescence from a Single Polymer: A Blue- Emitting Polyfluorene Incorporating Orange-Emitting Benzoselenadiazole Segments on Its Main Chain
}

\author{
CHEN-HAN CHIEN, PING-I SHIH, CHING-FONG SHU \\ Department of Applied Chemistry, National Chiao Tung University, 300, Hsinchu, Taiwan, Republic of China
}

Received 9 January 2007; accepted 14 February 2007

DOI: 10.1002 / pola. 22049

Published online in Wiley InterScience (www.interscience.wiley.com).

\begin{abstract}
We have developed efficient white-light-emitting polymers through the incorporation of low-bandgap orange-light-emitting benzoselenadiazole (BSeD) moieties into the backbone of a blue-light-emitting bipolar polyfluorene (PF) copolymer, which contains hole-transporting triphenylamine and electron-transporting oxadiazole pendent groups. By carefully controlling the concentrations of the low-energy-emitting species in the resulting copolymers, partial energy transfer from the blue-fluorescent $\mathrm{PF}$ backbone to the orange-fluorescent segments led to a single polymer emitting white light and exhibiting two balanced blue and orange emissions simultaneously. Efficient polymer light-emitting devices prepared using this copolymer exhibited luminance efficiencies as high as $4.1 \mathrm{~cd} / \mathrm{A}$ with color coordinates $(0.30,0.36)$ located in the white-light region. Moreover, the color coordinates remained almost unchanged over a range of operating potentials. A mechanistic study revealed that energy transfer from the PF backbone to the low-bandgap segments, rather than charge trapping, was the main operating process involved in the electroluminescence process. ๑ 2007 Wiley Periodicals, Inc. J Polym Sci Part A: Polym Chem 45: 2938-2946, 2007
\end{abstract}

Keywords: conjugated polymers; copolymerization; light-emitting diodes (LED)

\section{INTRODUCTION}

White polymer light-emitting diodes (WPLEDs) have attracted considerable attention because of their potential applications in solid state lighting and in back panel lighting for liquid crystal displays. ${ }^{1-3}$ In addition, PLEDs have the advantage of allowing large and flexible areas to be covered using solution processes, including spin-coating and ink-jet printing techniques, which are generally less expensive than the high-vacuum deposition techniques ${ }^{4-7}$ used commonly for small molecules. Generally, to achieve white emission from a PLED, it is desirable to utilize more than one active polymer because the emission of a single

Correspondence to: C.-F. Shu (E-mail: shu@cc.nctu.edu.tw) Journal of Polymer Science: Part A: Polymer Chemistry, Vol. 45, 2938-2946 (2007) @i)2007 Wiley Periodicals, Inc. polymer typically does not span the entire visible spectrum. Although several white-light-emitting single-polymer systems have been reported to exhibit blue emissions from the polymer itself and orange emissions from aggregating, excimer, or electroplex components, these systems have suffered from low efficiency. ${ }^{8-10}$ Various approaches toward realizing efficient WPLEDs have been described, ${ }^{11-17}$ especially for devices based on small-molecule-doped polymers or polymer-blend systems. More recently, single polymers emitting white light have been prepared through chemical incorporation of low-bandgap moieties into blueemitting polymers. ${ }^{18-23}$ Preparing WPLEDs from single polymers is desirable because it has advantages over blend systems in terms of avoiding both phase separation and complex fabrication processes. 
Polyfluorenes (PFs) are very promising candidates for blue-light-emitting materials because of their high photoluminescence (PL) and electroluminescence (EL) efficiencies and high thermal stabilities. ${ }^{24-27}$ In addition, PFs can be readily color-tuned through chemically or physically doping with lower-energy dyes. ${ }^{28-39}$ Consequently, PFs can function as both the host and the blue emitter in white-light-emitting devices. We have demonstrated that PF-TPA-OXD, a bipolar charge-transporting $\mathrm{PF}$ derivative containing hole-transporting triphenylamine (TPA) and electron-transporting oxadiazole (OXD) pendent groups, is not merely an efficient blue emitter with good spectral stability but also it is an ideal polymeric host for low-energy dopants. ${ }^{14,17,35,40}$ In this study, therefore, we incorporated orangeemitting benzoselenadiazole (BSeD) units covalently into the backbone of this dipolar PF copolymer with the aim of achieving white light emission from a single PF copolymer. By carefully controlling the concentrations of the low-energyemitting species in the resulting copolymers, partial energy transfer from the blue-fluorescent PF backbone to the orange-fluorescent segments resulted in a white-light-emitting single polymer exhibiting two balanced blue and orange emissions simultaneously. White EL was achieved, accompanied by a maximum luminance efficiency of $4.1 \mathrm{~cd} / \mathrm{A}$.

\section{EXPERIMENTAL}

\section{Materials}

Monomers 1-4, ${ }^{41-45}$ the model compound 4,7bis(9,9-dihexyl-2-fluorenyl)-2,1,3-benzoselenadiazole (BF-BSeD), ${ }^{16}$ and the blue-light-emitting polymer PF-TPA-OXD ${ }^{42}$ were prepared according to reported procedures. The solvents were dried using standard procedures. All other reagents were used as received from commercial sources unless otherwise stated.

\section{Characterization}

${ }^{1} \mathrm{H}$ and ${ }^{13} \mathrm{C}$ NMR spectra were recorded on Varian Unity $300 \mathrm{MHz}$ and Bruker-DRX $300 \mathrm{MHz}$ spectrometers. Mass spectra were obtained using a JEOL JMS-HX 110 mass spectrometer. Size exclusion chromatography was performed using a Waters chromatography unit interfaced to a Waters 410 differential refractometer; three 5- $\mu \mathrm{m}$ Waters styragel columns $\left(300 \times 7.8 \mathrm{~mm}^{2}\right)$ were DOI 10.1002/pola connected in series in order of decreasing pore size $\left(10^{4}, 10^{3}\right.$, and $10^{2} \AA$ ); tetrahydrofuran (THF) was the eluent. Standard polystyrene samples were used for calibration. Differential scanning calorimetry (DSC) was performed using a Seiko EXSTAR 6000 DSC unit operated at heating and cooling rates of 20 and $40{ }^{\circ} \mathrm{C} / \mathrm{min}$, respectively. Samples were scanned from 30 to $300{ }^{\circ} \mathrm{C}$, cooled to $0{ }^{\circ} \mathrm{C}$, and then scanned again from 30 to $300{ }^{\circ} \mathrm{C}$. The glass-transition temperatures $\left(T_{\mathrm{g}}\right)$ were determined from the second heating scan. Thermogravimetric analysis (TGA) was undertaken using a DuPont TGA 2950 instrument. The thermal stability of the samples under a nitrogen atmosphere was determined by measuring their weight loss while heating at a rate of $20^{\circ} \mathrm{C} / \mathrm{min}$. UV-Vis spectra were measured using an HP 8453 diode-array spectrophotometer. PL spectra were obtained using a Hitachi F-4500 luminescence spectrometer. Cyclic voltammetry (CV) measurements were performed using a BAS 100 B/W electrochemical analyzer operated at a scanning rate of $50 \mathrm{mV} / \mathrm{s}$; the supporting electrolyte was $0.1 \mathrm{M}$ tetrabutylammonium hexafluorophosphate $\left(\mathrm{TBAPF}_{6}\right)$ dissolved in anhydrous acetonitrile. The potentials were measured against an $\mathrm{Ag} / \mathrm{Ag}^{+}$ $\left(0.01 \mathrm{M} \mathrm{AgNO}{ }_{3}\right)$ reference electrode with ferrocene as the internal standard. The onset potentials were determined from the intersection of two tangents drawn at the rising and background currents of the cyclic voltammogram.

\section{Fabrication of LEDs}

We fabricated LED devices having the structure ITO/poly(styrenesulfonate)-doped poly(3,4-ethylenedioxythiophene) (PEDOT:PSS) (35 nm)/polymer emitting layer (50-70 nm)/TPBI $(30 \mathrm{~nm}) /$ Mg:Ag (100 nm)/Ag (100 nm). The PEDOT was spin-coated directly onto indium tin oxide (ITO) glass and dried at $80{ }^{\circ} \mathrm{C}$ for $12 \mathrm{~h}$ in vacuo to improve both the hole injection capability and the smoothness of the substrate. The light-emitting layer was spin-coated on top of the PEDOT layer using chlorobenzene as the solvent; the sample was then dried for $3 \mathrm{~h}$ at $60{ }^{\circ} \mathrm{C}$ in vacuo. Prior to film casting, the polymer solution was filtered through a Teflon filter $(0.45 \mu \mathrm{m})$. The TPBI layer, which we used as an electron transporting layer that would also block holes and confine excitons, was grown through thermal sublimation in a vacuum of $3 \times 10^{-6}$ torr. Subsequently, the cathode Mg:Ag (10:1, $100 \mathrm{~nm})$ alloy was deposited through coevaporation onto the TPBI layer; this process 
was followed by placing an additional layer of $\mathrm{Ag}(100 \mathrm{~nm})$ onto the alloy as a protection layer. The current-voltage-luminance relationships were measured under ambient conditions using a Keithley 2400 source meter and a Newport 1835C optical meter equipped with an 818ST silicon photodiode.

\section{General Polymerization Procedure}

Aqueous potassium carbonate $(2.0 \mathrm{M}, 0.72 \mathrm{~mL})$ and Aliquat $336(\sim 20 \mathrm{mg})$ were added to a mixture of monomers 1-4 in distilled toluene $(1.44 \mathrm{~mL})$. The mixture was degassed and then $\mathrm{Pd}\left(\mathrm{PPh}_{3}\right)_{4}(12 \mathrm{mg}, 5.5 \mathrm{~mol} \%)$ was added in one portion while flushing vigorously with nitrogen. The solution was heated at $110{ }^{\circ} \mathrm{C}$ for $24 \mathrm{~h}$ under nitrogen. The end groups were then capped by heating the mixture under reflux for $12 \mathrm{~h}$ with benzeneboronic acid and then for $12 \mathrm{~h}$ with bromobenzene. The reaction mixture was cooled to room temperature and the product was precipitated into a mixture of $\mathrm{MeOH}$ and $\mathrm{H}_{2} \mathrm{O}[3: 7(\mathrm{v} / \mathrm{v})$, $100 \mathrm{~mL}$. The crude polymer was collected and washed with excess $\mathrm{MeOH}$. The polymer was dissolved in THF, reprecipitated into $\mathrm{MeOH}$, washed with acetone for $48 \mathrm{~h}$ using a Soxhlet apparatus, and then dried under vacuum to afford the desired copolymers.

\section{TOF-BSeD 0.25}

A solution of monomers $\mathbf{1}(52.3 \mathrm{mg}, 59.7 \mu \mathrm{mol}), \mathbf{2}$ (61.8 mg, $59.7 \mu \mathrm{mol}), 3(500 \mu \mathrm{L}, 0.60 \mu \mathrm{mol}, 1.20$ $\times 10^{-3} \mathrm{M}$ solution in toluene), and $4(77.1 \mathrm{mg}$, $120 \mu \mathrm{mol})$ was copolymerized to yield TOFBSeD $_{\mathbf{0 . 2 5}}$ (106 mg, 83.5\%). ${ }^{1} \mathrm{H}$ NMR $(500 \mathrm{MHz}$, $\left.\mathrm{CDCl}_{3}\right): \delta 0.71-0.76(\mathrm{~m}, 20 \mathrm{H}), 0.89(\mathrm{t}, J=4.0 \mathrm{~Hz}$, $12 \mathrm{H}), 0.91(\mathrm{~m}, 40 \mathrm{H}), 1.28(\mathrm{~m}, 26 \mathrm{H}), 1.62(\mathrm{~m}, 8$ $\mathrm{H}), 2.05(\mathrm{~m}, 8 \mathrm{H}), 2.53(\mathrm{~m}, 8 \mathrm{H}), 6.91-7.18(\mathrm{~m}$, 24H), 7.55-7.86 (m, 30H), 7.97-8.11 (m, $10 \mathrm{H}) .{ }^{13} \mathrm{C}$ NMR (125 MHz, $\left.\mathrm{CDCl}_{3}\right): \delta 14.0,14.1,22.4,22.5$, $23.8,29.2,30.0,31.1,31.7,33.6,35.0,35.1,40.3$, $55.3,64.7,65.8,120.0,121.0,121.3,121.8,122.3$, $122.9,123.9,124.5,124.6,126.0,126.7,127.3$, $128.0,128.3,128.9,129.1,129.8,131.9,137.5$, $138.5,138.9,140.3,145.3,146.7,149.2,150.8$, 151.9, 152.8, 155.4, 164.0, 164.7. Anal. Calcd: C, 87.16; H, 7.91; N, 3.55. Found: C, 86.12; H, 7.72; $\mathrm{N}, 3.73$.

\section{TOF-BSeD 0.5}

A mixture of monomers $1(52.1 \mathrm{mg}, 59.4 \mu \mathrm{mol})$, 2 (61.5 mg, $59.4 \mu \mathrm{mol}), 3(200 \mu \mathrm{L}, 1.20 \mu \mathrm{mol}, 6.00$ $\times 10^{-4} \mathrm{M}$ solution in toluene), and $4(77.1 \mathrm{mg}$, $120 \mu \mathrm{mol}$ ) was copolymerized to yield TOFBSeD $_{0.5}$ (115 mg, 75.9\%). TOF-BSeD 0.5 exhibited virtually identical ${ }^{1} \mathrm{H}$ and ${ }^{13} \mathrm{C}$ NMR spectra as those of TOF-BSeD $\mathbf{0 . 2 5}_{\mathbf{0}}$ because these two copolymers had very similar chemical structures and compositions.

\section{TOF-BSeD 0.7}

A solution of monomers $\mathbf{1}(49.3 \mathrm{mg}, 56.2 \mu \mathrm{mol}), \mathbf{2}$ $(58.2 \mathrm{mg}, 56.2 \mu \mathrm{mol}), \mathbf{3}(200 \mu \mathrm{L}, 1.60 \mu \mathrm{mol}, 8.00$ $\times 10^{-3} \mathrm{M}$ solution in toluene), and $4(73.3 \mathrm{mg}$, $114 \mu \mathrm{mol})$ was copolymerized to yield TOFBSeD $_{0.7}$ (106 mg, 81.2\%). TOF-BSeD $\mathbf{0 . 7}_{\text {.7 }}$ exhibited virtually identical ${ }^{1} \mathrm{H}$ and ${ }^{13} \mathrm{C}$ NMR spectra as those of TOF-BSeD $\mathbf{D}_{\mathbf{0 . 2 5}}$ because these two copolymers had very similar chemical structures and compositions.

\section{RESULTS AND DISCUSSION}

\section{Synthesis}

Scheme 1 illustrates the synthetic route followed for the preparation of TOF-BSeD $\mathbf{D}_{\boldsymbol{x}}$ copolymers, including the feed ratios of the comonomers. The OXD-containing monomer $1,{ }^{41}$ TPA-containing monomer $2{ }^{42}$ 4,7-dibromo-2,1,3-benzoselenadiazole (3), ${ }^{43,44}$ and the diboronate $4^{45}$ were synthesized according to reported procedures. The

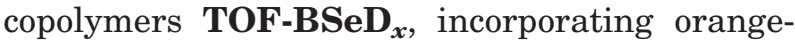
light-emitting BSeD units in the PF main chain, were synthesized through Suzuki coupling between the dibromides 1-3 and the diboronate 4. To fine-tune the relative intensity of the blue and orange emissions, the content of BSeD units in the copolymers was controlled within the range from 0.25 to $0.70 \mathrm{~mol} \%$. The copolymerizations were performed using $\mathrm{Pd}\left(\mathrm{PPh}_{3}\right)_{4}$ as the catalyst and Aliquat 336 as the phase-transfer reagent in a mixture of toluene and aqueous $\mathrm{K}_{2} \mathrm{CO}_{3}(2.0 \mathrm{M})$. When the polymerizations were complete, the end groups of the polymer chain were capped by heating the mixture under reflux sequentially with phenylboronic acid and bromobenzene. The resultant polymers were random copolymers possessing backbones consisting of fluorene segments of different lengths separated by single BSeD units at both ends of each segment. The selenium content in TOF-BSeD T $_{\boldsymbol{x}}(0.033-0.094 \mathrm{wt} \%)$, as measured using inductively coupled plasma mass spectrometry (ICP-MS), matched the monomer feed ratio agreeably $(0.029-0.105$ wt \%).

Journal of Polymer Science: Part A: Polymer Chemistry DOI 10.1002/pola 


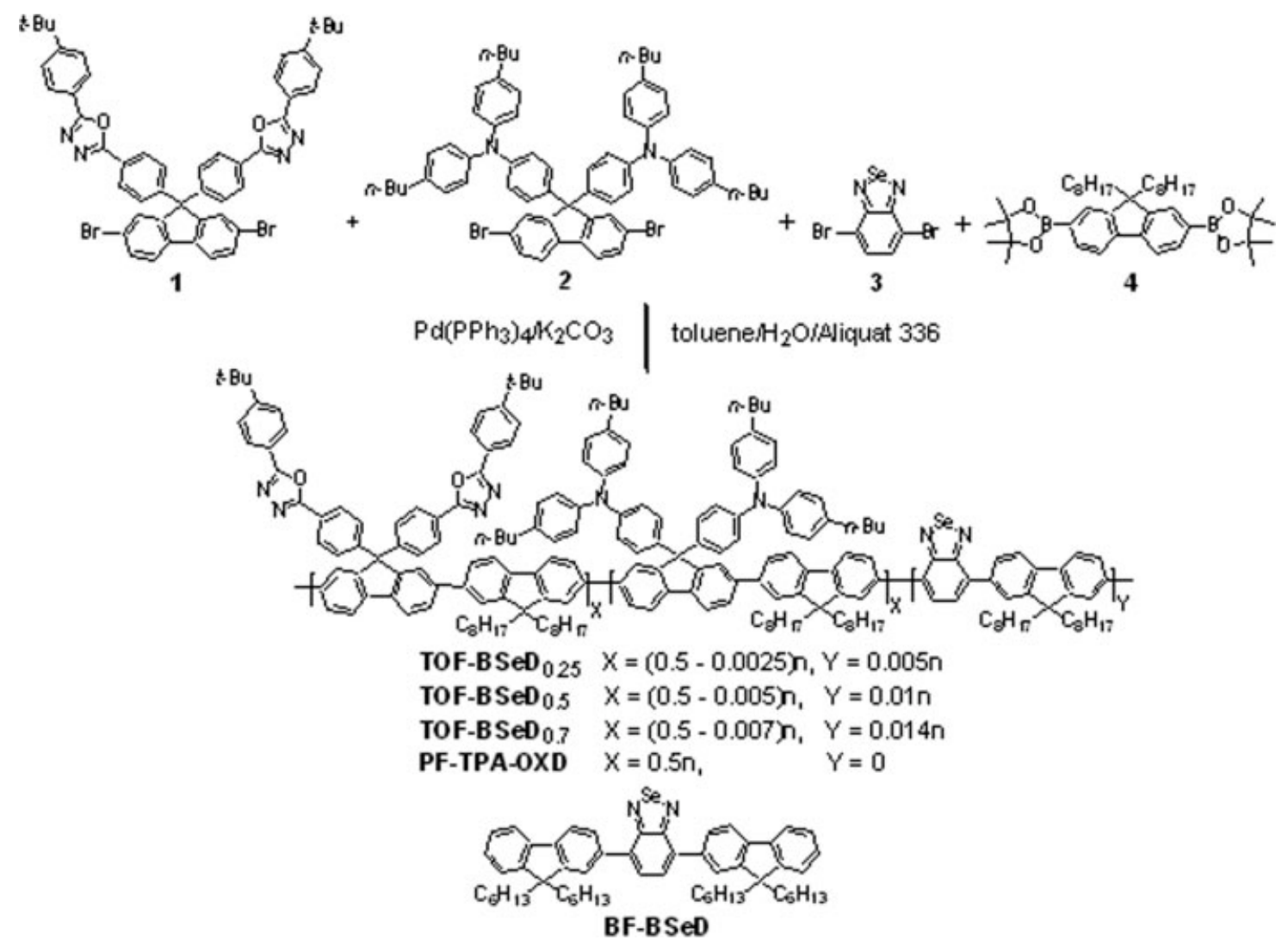

Scheme 1. Synthesis of PF copolymers and chemical structure of orange-emitting model compound.

The TOF-BSeD $\mathbf{D}_{\boldsymbol{x}}$ copolymers readily dissolved in common organic solvents, such as toluene, chlorobenzene, chloroform, and THF. Their number-average molecular weights $\left(M_{\mathrm{n}}\right)$, determined using gel permeation chromatography with polystyrenes as standards, were in the range 3.1-5.8 $\times 10^{4} \mathrm{~g} / \mathrm{mol}$ with polydispersities ranging from 1.7 to 1.9 . We investigated the thermal properties of these copolymers using TGA and DSC. All the polymers exhibited good thermal properties, with $5 \%$ weight losses occurring above $400{ }^{\circ} \mathrm{C}$ and glass transition temperatures $\left(T_{\mathrm{g}}\right)$ at $\sim 180{ }^{\circ} \mathrm{C}$. Such relatively high-temperature glass transitions can prevent morphological changes from occurring upon exposure to heat; this characteristic is an essential one for polymers that are to be used as emissive materials for light-emitting applications.

\section{Photophysical Properties}

Figures 1 and 2 display the absorption and PL spectra of TOF-BSeD $\boldsymbol{x}_{\boldsymbol{x}}$ in dilute THF solutions and in solid films; for the sake of comparison, the absorption and PL spectra of PF-TPA-OXD are also provided. The absorption spectra of TOFBSeD $_{\boldsymbol{x}}$ are similar to those of PF-TPA-OXD, both in solution and in the solid state, with two major absorptions appearing at $\sim 300$ and $390 \mathrm{~nm}$. The first absorption in the short-wavelength region originates from the combined absorptions of the TPA and OXD pendent groups; the second arises from $\pi-\pi^{*}$ transitions of the conjugated PF backbone. There was no detectable absorption band for the BSeD units (at $\sim 440 \mathrm{~nm}$ for the model compound BF-BSeD, Fig. 3) because of their very low abundances in these copolymers. For the same reason, the PL spectra of TOF$\mathbf{B S e D}_{\boldsymbol{x}}$ in dilute solutions displayed two vibronic peaks (at 420 and $444 \mathrm{~nm}$ ) that are identical to those observed in the PL spectrum of PF-TPAOXD; i.e., the spectral features represented only the polyfluorenyl chromophoric segments. In contrast, the incorporation of BSeD units into the main chain greatly enhanced the luminescence properties in the thin films. As indicated in Figure 2(b), the PL spectra of TOF-BSeD films $_{\boldsymbol{x}}$ spin-coated onto quartz substrates contain, in 


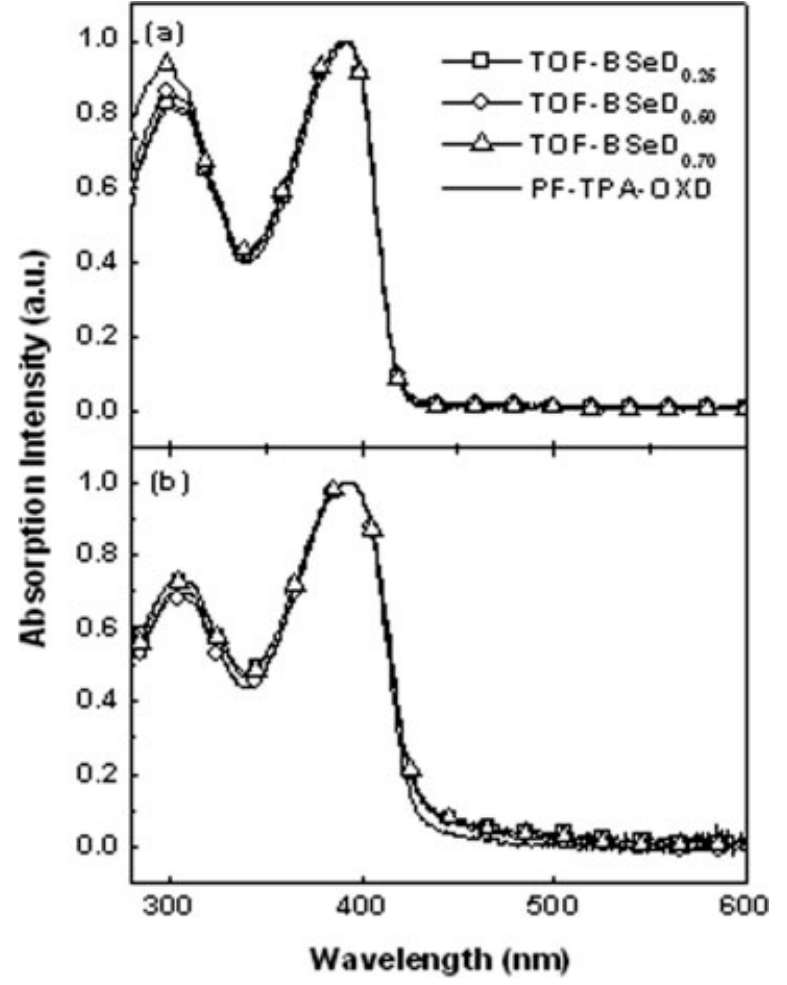

Figure 1. UV-vis absorption spectra of the copolymers (a) in dilute THF solutions and (b) in the solid state.

addition to their PF emissions, a longer-wavelength emission band observed in the orange region arising from the narrow-bandgap BSeD units. To further understand the interactions between the fluorene segments and the lowenergy-emitting species, we prepared a model compound, BF-BSeD, and investigated the optical behavior of the orange-emitting moieties in the backbones of the dye-attached copolymers. Figure 3 indicates that the absorption spectrum of BF-BSeD overlaps well with the emission spectrum of $\mathrm{PF}$, implying that efficient energy transfer occurred from the fluorene segments to the low-energy-emitting moieties in the dyeattached copolymers. The enhanced orange emission intensities in the solid state PL spectra presented in Figure 2(b) indicate that energy transfer from the fluorene segments to the low-energy-emitting segments was facilitated through both intra- and interchain channels because of the shorter distances between the polymer chains in the solid state. With the increase of the content of BSeD unit, the contribution of the orange emission in Figure 2(b) enlarges. In the case of TOF-BSeD $\mathbf{D . 7}_{\mathbf{0 . 7}}$, this copolymer exhibits relatively balanced intensities of the blue and

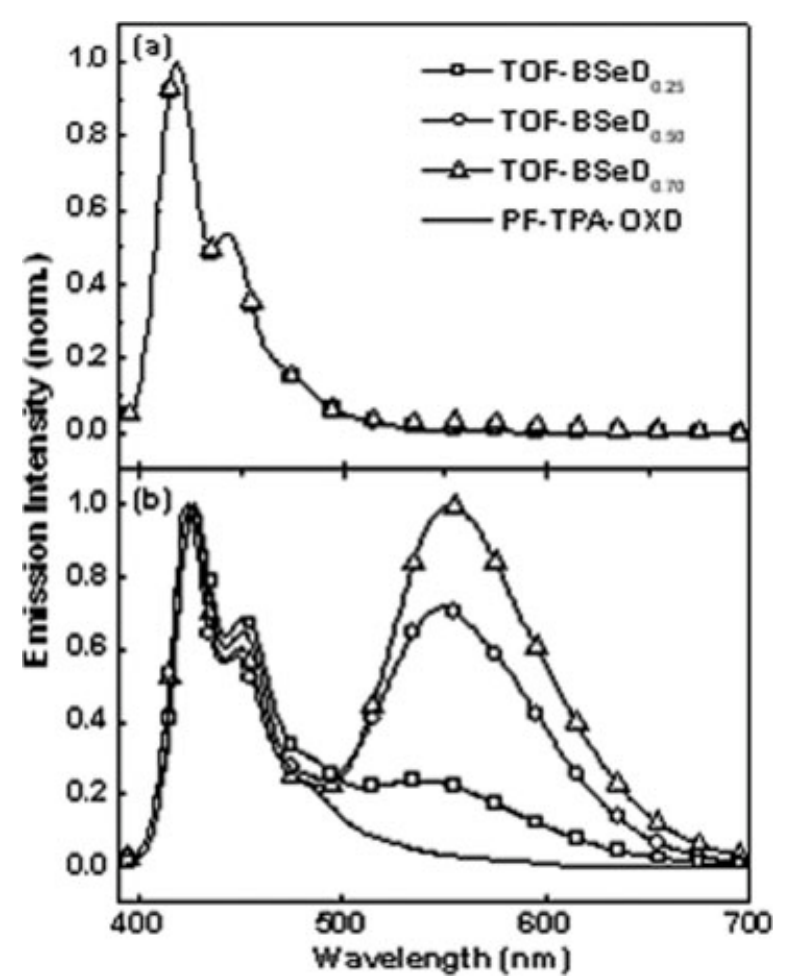

Figure 2. PL spectra of the copolymers (a) in dilute THF solutions and (b) in the solid state.

orange emissions, resulting in white emission (vide post).

Furthermore, to investigate the energy transfer between the pendent TPA and OXD groups and the main chain, the solid films were also excited at $300 \mathrm{~nm}$, i.e., the absorption maxima of the side chains. We found that the PL spectra

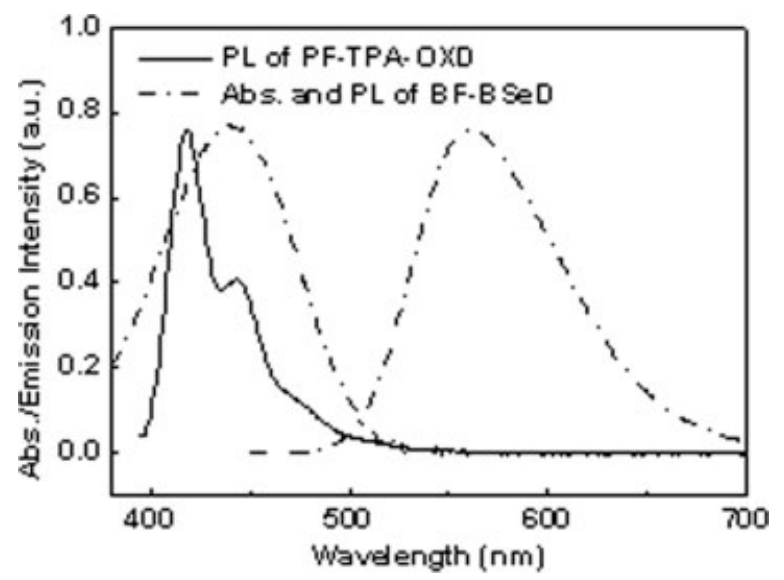

Figure 3. PL spectrum of PF-TPA-OXD (excited at $390 \mathrm{~nm}$ ) in the solid state and the PL and absorption spectra of BF-BSeD (excited at $440 \mathrm{~nm}$ ) in dilute THF solution.

Journal of Polymer Science: Part A: Polymer Chemistry DOI 10.1002/pola 


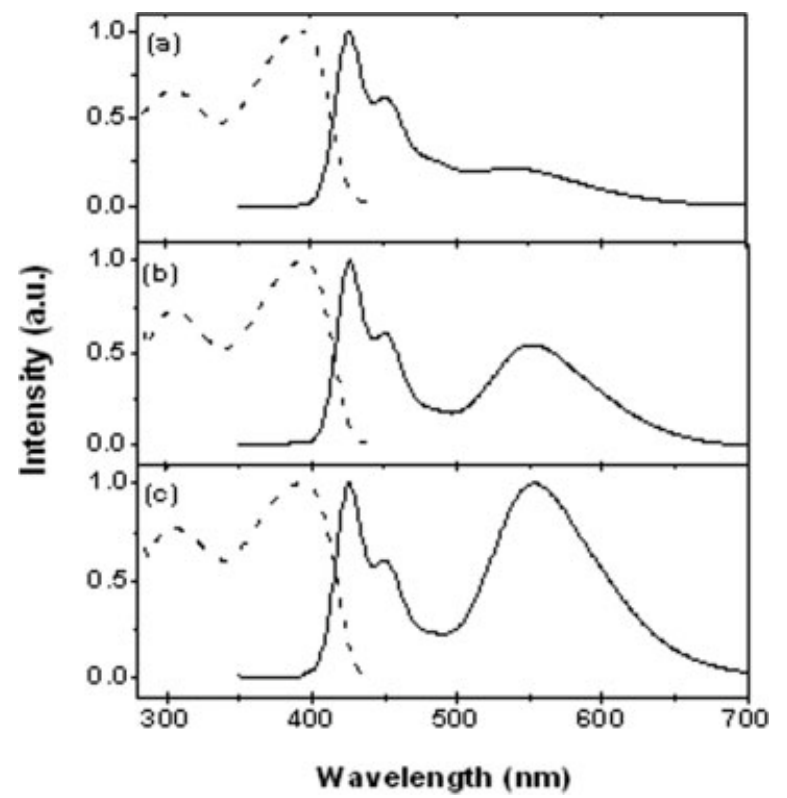

Figure 4. PL (solid lines, excited at $300 \mathrm{~nm}$ ) and excitation (dashed lines, monitored emission at $550 \mathrm{~nm}$ ) spectra of (a) TOF-BSeD $\mathbf{0 . 2 5}_{\text {, (b) TOF-BSeD }}$, , and (c) TOF-BSeD $\mathbf{D}_{\mathbf{7}}$ in the solid state.

superimposed well with that obtained under excitation of the PF segments at $390 \mathrm{~nm}$ (Fig. 4). No luminescence was detectable at $\sim 360 \mathrm{~nm}$ from either the TPA or OXD side chains. Moreover, the excitation spectra, monitored at $550 \mathrm{~nm}$, were almost identical to the absorption spectra. These results reveal that, in addition to direct energy transfer from the excited polyfluorenyl segments to the doped BSeD units, a cascade energy transfer, mediated by the PF backbone, occurred from the excited TPA and OXD pendent groups to the lower-energy BSeD sites.

\section{Electrochemical Properties}

We employed CV to investigate the redox behavior of the copolymers and to estimate their HOMO and LUMO energy levels. The electrochemical behavior of the polymer films coated onto glassy carbon electrodes was studied using an electrolyte of $0.1 \mathrm{M} \mathrm{TBAPF}{ }_{6}$ in acetonitrile; ferrocene was employed as the internal standard. TOF-BSeD ${ }_{x}$ exhibited similar redox behavior to that of PF-TPA-OXD, with onset potentials for oxidation and reduction at 0.50 and $-2.30 \mathrm{~V}$, respectively. In addition, we did not observe any electrochemical behavior attributable to the

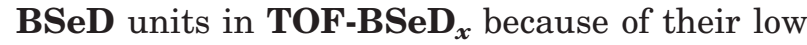
contents in the polymer backbones. These results indicate that the introduction of a small number of orange-emitting moieties did not affect the electrochemical properties of the original polymers. Similar phenomena have been observed from studies of related fluorene-based copolymers. ${ }^{16,22}$ Based on the onset potentials, we estimated the HOMO and LUMO energy levels of these three copolymers to be -5.30 and $-2.50 \mathrm{eV}$, respectively, in relation to the energy level of the ferrocene reference ( $4.8 \mathrm{eV}$ below the vacuum level). ${ }^{46}$

\section{EL Properties}

To evaluate the EL properties of the polymers, we fabricated devices having the configuration ITO/ PEDOT $(35 \mathrm{~nm}) /$ TOF-BSeD $\boldsymbol{x}_{\boldsymbol{x}}(50-70 \mathrm{~nm}) / \mathrm{TPBI}$ $(30 \mathrm{~nm}) / \mathrm{Mg}: \mathrm{Ag}(100 \mathrm{~nm}) / \mathrm{Ag}(100 \mathrm{~nm})$. The TPBI layer was employed as an electron-transporting layer that would also block holes and confine excitons. As indicated in Figure 5, the EL spectra of the three polymers display dual emissions comprising both blue emissions (at $\sim 430$ and $450 \mathrm{~nm}$ ) from the polyfluorenyl segments and orange emissions (at $\sim 550 \mathrm{~nm}$ ) from the BSeD units. Because the EL spectra of TOF-BSeD $\mathbf{D}_{\boldsymbol{x}}$ are quite similar to their corresponding PL spectra presented in Figure 2(b), energy transfer from the fluorene segments to the BSeD-based units is probably the main operating mechanism involved in the EL process. ${ }^{47,48}$ Figure 6 presents an energy level diagram, based on TOF-BSeD $\boldsymbol{D}_{\boldsymbol{x}}$ and the model compound BF-BSeD ${ }^{16}$ that we constructed to obtain information regarding the charge-transporting mechanism in the EL devices. In our device configuration, holes would be

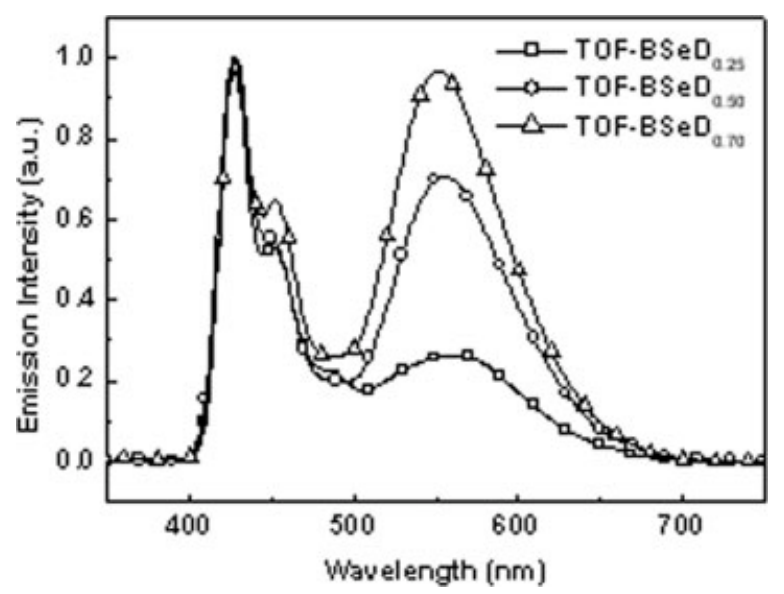

Figure 5. EL spectra of the ITO/PEDOT/TOF$\mathbf{B S e D}_{\boldsymbol{x}} / \mathrm{TPBI} / \mathrm{Mg}: \mathrm{Ag}$ devices recorded at an applied potential of $9 \mathrm{~V}$. 


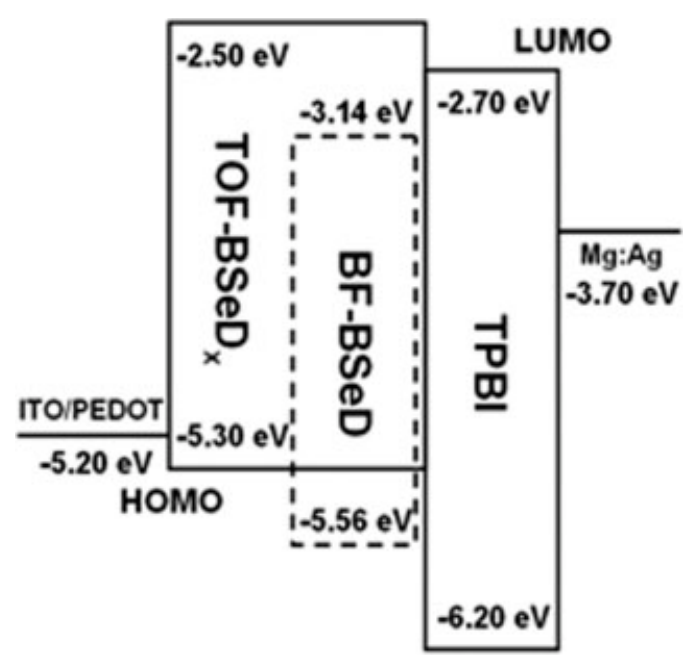

Figure 6. Energy level diagram for the materials involved in EL devices having the configuration ITO/ PEDOT/polymer/TPBI/Mg:Ag.

the majority carriers because of the relatively lower injection barrier $(0.1 \mathrm{eV})$ from the ITO/ PEDOT electrode to the next organic layer (TOF$\mathbf{B S e D}_{\boldsymbol{x}}$ ). The energy diagram suggests that the BSeD units could not work as trapping sites for holes, the major carriers, and that the charge trapping mechanism is insignificant in the TOF$\mathbf{B S e D}_{\boldsymbol{x}}$-based devices. As a result, we observed very few differences between the PL and EL spectra of these polymers. This finding is consistent with our previous observations of only slight differences between the EL and PL spectra of the individual polymers. ${ }^{16}$

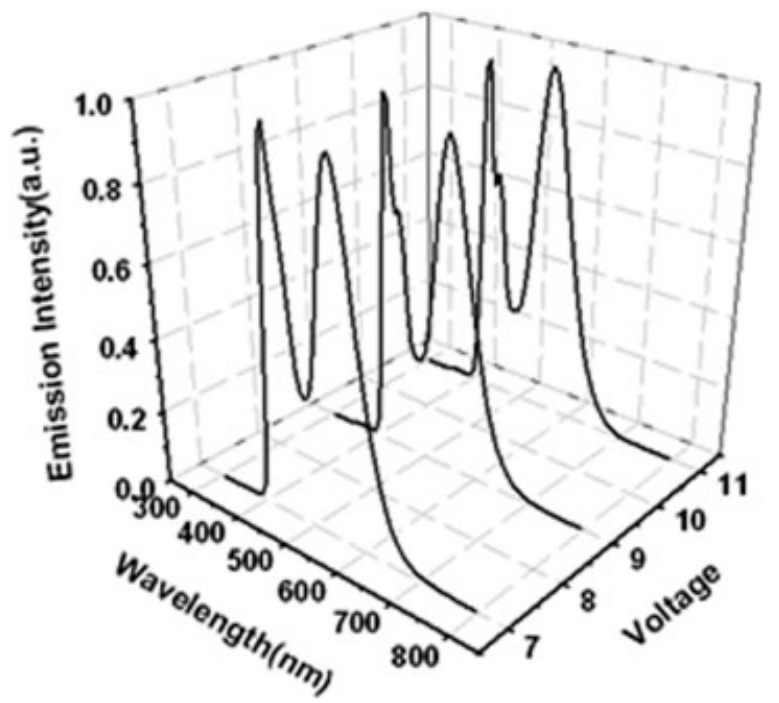

Figure 7. EL spectra of the device based on TOF$\mathbf{B S e D}_{\mathbf{0 . 7}}$ operated at various driving potentials.

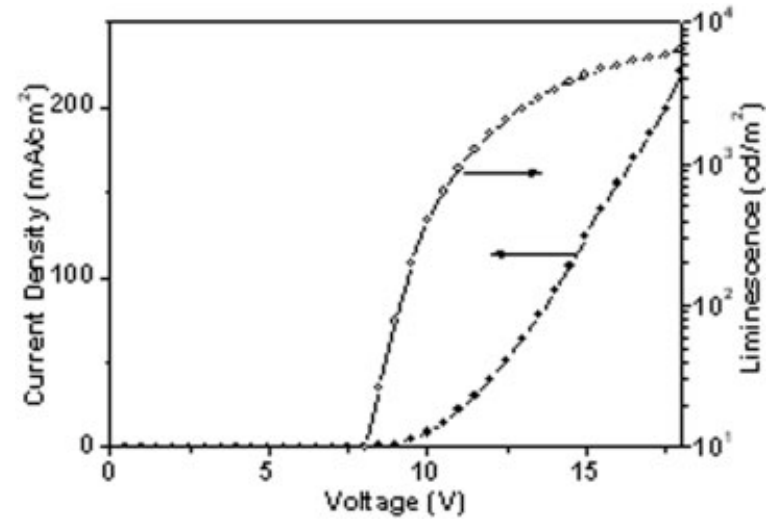

Figure 8. Current density-voltage-luminance characteristics of the TOF-BSeD $\mathbf{D}_{\mathbf{0 . 7}}$-based device.

Among the EL spectral emissions of the three copolymers, the emission of TOF-BSeD $\mathbf{D}_{\mathbf{0 . 7}}$ was especially wide, covering the entire visible region (400-700 $\mathrm{nm}$ ) with relatively balanced intensities of the blue and orange emissions, resulting in an apparent white color. The Commission Interationale d'Enclairage (CIE) chromaticity coordinates of the device were $(0.30,0.36)$ at a bias of $9 \mathrm{~V}$, quite close to the equienergy white point $(0.33$, 0.33). Moreover, the emission color remained almost constant under different bias conditions. As indicated in Figure 7, the EL spectra of the TOF-BSeD $\mathbf{0 . 7}_{\text {.7 }}$-based device remained practically unchanged when the bias was increased from 7 to $11 \mathrm{~V}$. In contrast, the emissions of white-light OLEDs based on polymer blend systems and single polymers containing small-molecule dyes are typically sensitive to the driving voltage, probably because of phase separation or dye aggregation. ${ }^{22,49,50}$ The bias-independent white emission exhibited by our single-polymer system, in which the chromophore units are dispersed covalently within the polymer matrix, is desirable for display or illumination applications. Figure 8 displays the current density-voltage-luminance $(I-V$ $L)$ curves of the TOF-BSeD $\mathbf{0 . 7}_{\mathbf{0}}$-based white-lightemitting devices. The device was turned on at an applied potential of $6.7 \mathrm{~V}$ (corresponding to $1 \mathrm{~cd} /$ $\mathrm{m}^{2}$ ) and a brightness of $10^{3} \mathrm{~cd} / \mathrm{m}^{2}$ was achieved at $\sim 11$ V. As plotted in Figure 9, the maximum luminance efficiency of white EL was $4.1 \mathrm{~cd} / \mathrm{A}$, corresponding to an external quantum efficiency of $1.5 \%$, obtained at a bias of $10 \mathrm{~V}$ and a brightness of $394 \mathrm{~cd} / \mathrm{m}^{2}$. Even when the luminance in our white-light-emitting device was increased to the order of $10^{3} \mathrm{~cd} / \mathrm{m}^{2}$ (at $\sim 27 \mathrm{~mA} / \mathrm{cm}^{2}$ ), the corresponding EL efficiency was virtually unchanged at $3.9 \mathrm{~cd} / \mathrm{A}$.

Journal of Polymer Science: Part A: Polymer Chemistry 


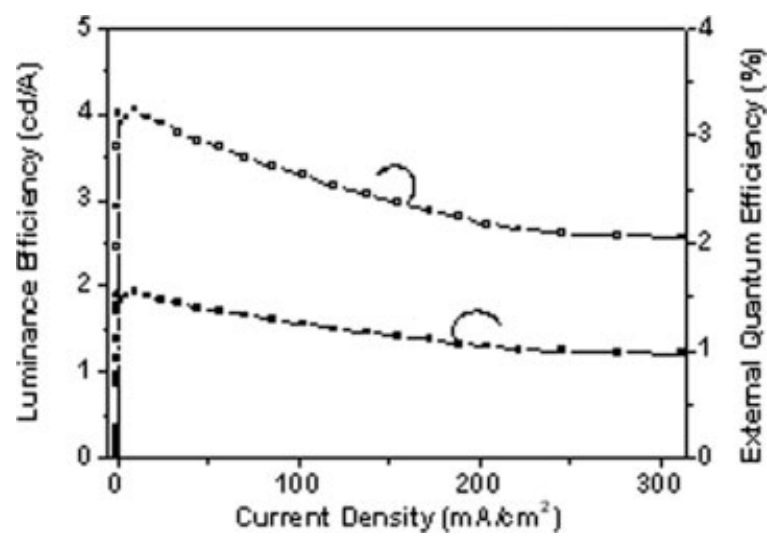

Figure 9. External quantum efficiency and luminance efficiency of the device based on TOF-BSeD $\mathbf{D . 7}_{\mathbf{0}}$, plotted as functions of the current density.

\section{CONCLUSIONS}

We have developed a single polymer that emits white light by incorporating small amounts of orange-light-emitting benzoselenadiazole moieties into the backbone of a bipolar blue-light-emitting $\mathrm{PF}$. As a result of partial energy transfer from the blue-fluorescent $\mathrm{PF}$ to the orange-fluorescent component, the copolymers TOF-BSeD ${ }_{x}$ exhibit dual emissions comprising the individual emissions of the two emissive species. Through tuning of the content of BSeD, white-light emission was realized in the TOF-BSeD $\mathbf{D . 7}_{\mathbf{7}}$ system. Efficient polymer light-emitting devices prepared using this copolymer exhibited luminance efficiencies as high as $4.1 \mathrm{~cd} / \mathrm{A}$ with CIE coordinates $(0.30$, 0.36 ) located in the white-light region. Even when the luminance in the white-light-emitting device was increased to the order of $10^{3} \mathrm{~cd} / \mathrm{m}^{2}$ (at $\sim 27$ $\mathrm{mA} / \mathrm{cm}^{2}$ ), the corresponding EL efficiency still maintained at $3.9 \mathrm{~cd} / \mathrm{A}$. Moreover, because phase separation did not occur in this covalently doped single-polymer system, the color coordinates remained almost unchanged upon varying the operating potential.

We thank the National Science Council and the MOE ATU Program for financial support. We are especially grateful to Professor C.-H. Cheng for his support and cooperation during the preparation and characterization of the light-emitting devices.

\section{REFERENCES AND NOTES}

1. Kido, J.; Kimura, M.; Nagai, K. Science 1995, 267, 1332.

Journal of Polymer Science: Part A: Polymer Chemistry DOI 10.1002/pola
2. Kido, J.; Shionoya, H.; Nagai, K. Appl Phys Lett 1995, 67, 2281.

3. Tasch, S.; List, E. J. W.; Ekström, O.; Graupner, W.; Leising, G.; Schlichting, P.; Rohr, U.; Geerts, Y.; Scherf, U.; Müllen, K. Appl Phys Lett 1997, 71, 2883.

4. Kraft, A.; Grimsdale, A. C.; Holmes, A. B. Angew Chem Int Ed Engl 1998, 37, 402.

5. Friend, R. H.; Gymer, R. W.; Holmes, A. B.; Burroughes, J. H.; Marks, R. N.; Taliani, C.; Bradley, D. D. C.; Dos Santos, D. A.; Brédas, J. L.; Lögdlund, M.; Salaneck, W. R. Nature 1999, 397, 121.

6. Bernius, M. T.; Inbasekaran, M.; O’Brien, J.; Wu, W. Adv Mater 2000, 12, 1737.

7. Akcelrud, L. Prog Polym Sci 2003, 28, 875.

8. Paik, K. L.; Baek, N. S.; Kim, H. K.; Lee, J.-H.; Lee, Y. Macromolecules 2002, 35, 6782.

9. Tsai, M.-L.; Liu, C.-Y.; Hsu, M.-A.; Chow, T. J. Appl Phys Lett 2003, 82, 550.

10. Furuta, P. T.; Deng, L.; Garon, S.; Thompson, M. E.; Fréchet, J. M. J. J Am Chem Soc 2004, 126, 15388.

11. Hu, B.; Karasz, F. E. J Appl Phys 2003, 93, 1995.

12. Xu, Q.; Duong, H. M.; Wudl, F.; Yang, Y. Appl Phys Lett 2004, 85, 3357.

13. Su, H.-J.; Wu, F.-I.; Shu, C.-F. Macromolecules 2004, 37, 7197.

14. Kim, J. H.; Herguth, P.; Kang, M.-S.; Jen, A. K.-Y.; Tseng, Y.-H.; Shu, C.-F. Appl Phys Lett 2004, 85, 1116.

15. Gong, X.; Ma, W.; Ostrowski, J. C.; Bazan, G. C.; Moses, D.; Heeger, A. J. Adv Mater 2004, 16, 615.

16. Shih, P.-I.; Tseng, Y.-H.; Wu, F.-I.; Dixit, A. K.; Shu, C.-F. Adv Funct Mater 2006, 16, 1582.

17. Wu, F.-I.; Shih, P.-I.; Tseng, Y.-H.; Shu, C.-F.; Tung, Y.-L.; Chi, Y. J Mater Chem 2007, 17, 167.

18. Tu, G.; Zhou, Q.; Cheng, Y.; Wang, L.; Ma, D.; Jing, X.; Wang, F. Appl Phys Lett 2004, 85, 2172.

19. Lee, S. K.; Hwang, D.-H.; Jung, B.-J.; Cho, N. S.; Lee, J.; Lee, J.-D.; Shim, H.-K. Adv Funct Mater 2005, 15, 1647.

20. Liu, J.; Zhou, Q. G.; Cheng, Y. X.; Geng, Y. H.; Wang, L. X.; Ma, D. G.; Jing, X. B.; Wang, F. S. Adv Mater 2005, 17, 2974.

21. Tu, G. L.; Mei, C. Y.; Zhou, Q. G.; Cheng, Y. X.; Geng, Y. H.; Wang, L. X.; Ma, D. G.; Jing, X. B.; Wang, F. S. Adv Funct Mater 2006, 16, 101.

22. Liu, J.; Zhou, Q. G.; Cheng, Y. X.; Geng, Y. H.; Wang, L. X.; Ma, D. G.; Jing, X. B.; Wang, F. S. Adv Funct Mater 2006, 16, 957.

23. Jiang, J. X.; Xu, Y. H.; Yang, W.; Guan, R.; Liu, Z. Q.; Zhen, H. Y.; Cao, Y. Adv Mater 2006, 18, 1769 .

24. Leclerc, M. J Polym Sci Part A: Polym Chem 2001, 39, 2867.

25. Grigalevicius, S.; Ma, L.; Xie, Z.-Y.; Scherf, U. J Polym Sci Part A: Polym Chem 2006, 44, 5987.

26. Wu, C.-W.; Sung, H.-H.; Lin, H.-C. J Polym Sci Part A: Polym Chem 2006, 44, 6765. 
27. Ashraf, R. S.; Hoppe, H.; Shahid, M.; Gobsch, G.; Sensfuss, S.; Klemm, E. J Polym Sci Part A: Polym Chem 2006, 44, 6952.

28. Hou, Q.; Xu, Y.; Yang, W.; Yuan, M.; Peng, J.; Cao, Y. J Mater Chem 2002, 12, 2887.

29. Herguth, P.; Jiang, X.; Liu, M. S.; Jen, A. K.-Y. Macromolecules 2002, 35, 6094.

30. Chen, X.; Liao, J.-L.; Liang, Y.; Ahmed, M. O.; Tseng, H.-E.; Chen, S.-A. J Am Chem Soc 2003, 125,636 .

31. Sandee, A. J.; Williams, C. K.; Evans, N. R.; Davies, J. E.; Boothby, C. E.; Köhler, A.; Friend, R. H.; Holmes, A. B. J Am Chem Soc 2004, 126, 7041.

32. Yang, R.; Tian, R.; Yan, J.; Zhang, Y.; Yang, J.; Hou, Q.; Yang, W.; Zhang, C.; Cao, Y. Macromolecules 2005, 38, 244.

33. Su, H.-J.; Wu, F.-I.; Tseng, Y.-H.; Shu, C.-F. Adv Funct Mater 2005, 15, 1209.

34. Su, H.-J.; Wu, F.-I.; Shu, C.-F.; Tung, Y.-L.; Chi, Y.; Lee, G.-H. J Polym Sci Part A: Polym Chem 2005, 43, 859

35. Wu, F.-I.; Shih, P.-I.; Tseng, Y.-H.; Chen, G.-Y.; Chien, C.-H.; Shu, C.-F. J Phys Chem B 2005, 109, 14000.

36. Lim, E.; Jung, B.-J.; Shim, H.-K. J Polym Sci Part A: Polym Chem 2006, 44, 243.

37. Huang, F.; Hou, L.; Shen, H.; Yang, R.; Hou, Q.; Cao, Y. J Polym Sci Part A: Polym Chem 2006, 44, 2521.
38. Kim, J. H.; You, N.-H.; Lee, H. J Polym Sci Part A: Polym Chem 2006, 44, 3729.

39. Luo, J.; Hou, Q.; Chen, J.; Cao, Y. Synth Met 2006, 156,470 .

40. Niu, Y.-H.; Tung, Y.-L.; Chi, Y.; Shu, C.-F.; Kim, J. H.; Chen, B.; Luo, J.; Carty, A. J.; Jen, A. K.-Y. Chem Mater 2005, 17, 3532.

41. Wu, F.-I.; Reddy, D. S.; Shu, C.-F.; Liu, M. S.; Jen, A. K.-Y. Chem Mater 2003, 15, 269.

42. Shu, C.-F.; Dodda, R.; Wu, F.-I.; Liu, M. S.; Jen, A. K.-Y. Macromolecules 2003, 36, 6698.

43. Bird, C. W.; Cheeseman, G. W. H.; Sarsfield, A. A. J Chem Soc 1963, 4767.

44. Yang, R.; Tian, R.; Hou, Q.; Yang, W.; Cao, Y. Macromolecules 2003, 36, 7453.

45. Ranger, M.; Rondeau, D.; Leclerc, M. Macromolecules 1997, 30, 7686.

46. Pommerehne, J.; Vestweber, H.; Guss, W.; Mahrt, R. F.; Bässler, H.; Porsch, M.; Daub, J. Adv Mater 1995, 7, 551.

47. Uchida, M.; Adachi, C.; Koyama, T.; Taniguchi, Y. J Appl Phys 1999, 86, 1680.

48. Virgili, T.; Lidzey, D. C.; Bradley, D. D. C. Synth Met 2000, 111, 203.

49. Alam, M. M.; Tonzola, C. J.; Jenekhe, S. A. Macromolecules 2003, 36, 6577.

50. Ananthakrishnan, N.; Padmanaban, G.; Ramakrishnan, S.; Reynolds, J. R. Macromolecules 2005, 38, 7660 . 